\title{
METABOLISMO RESPIRATÓRIO EM RELAÇÃO À TEMPERATURA E FOTOPERÍODO EM Oxidus gracilis (Diplopoda, Polydesmida, Strongylosomidae)
}

\section{OXYGEN CONSUMPTION RELATED TO TEMPERATURE AND PHOTOPERIOD IN Oxidus gracilis (Diplopoda, Polydesmida, Strongylosomidae)}

\section{Silva, R.S. ${ }^{1}$, Penteado, C.H.S. ${ }^{2}$; Boccardo, L. ${ }^{3}$}

${ }^{1}$ ESALQ-USP - C.P. 56 - Piracicaba, SP. Brasil - CEP 13400-970

${ }^{2}$ Pesquisador Voluntário do Centro de Estudos Ambientais - CEA/UNESP - Campus de Rio Claro, Av. 24A, 1515 - CEP-13506-900 - Rio Claro, SP, Brasil.

${ }^{3}$ Departamento de Ciências Biológicas, Universidade Estadual do Sudoeste da Bahia, UESB - Campus de Jequié, Av. José Moreira Sobrinho, S/N, CEP-45200-000 - Jequié, BA, Brasil.

\section{RESUMO}

O metabolismo respiratório do diplópodo Oxidus gracilis foi pesquisado tendo como referência os parâmetros temperatura e fotoperíodo, buscando, no primeiro caso, detectar eventuais ajustes metabólicos compensatórios para variações da temperatura e, no segundo, as alterações decorrentes do ritmo de atividade no ciclo de 24 horas. Nos dois casos foi empregado o microrespirômetro de Warburg, usando-se frascos de $20 \mathrm{ml}$ de capacidade volumétrica, aos quais foram adicionados $0,3 \mathrm{ml}$ de solução de $\mathrm{KOH}$ a $14 \%$ para a absorção do $\mathrm{CO}_{2}$ expirado. Os testes com temperatura foram feitos a $5^{\circ}, 10^{\circ}, 15^{\circ}, 20^{\circ}, 25^{\circ} 30^{\circ}, 35^{\circ}$ e $40^{\circ} \mathrm{C}$ e os de ritmos metabólicos foram feitos somente a $25^{\circ} \mathrm{C}$, durante 24 horas; com medidas respirométricas a cada 3 horas. Os dados foram apresentados como taxas respiratórias traduzidas em $\mathrm{m}^{1} \mathrm{O}_{2} \cdot \mathrm{g}\left(\mathrm{vivo}^{-1}\right)^{-1}$ hora $^{-1}$. Os resultados indicaram a ocorrência de uma estabilização metabólica a na curva R-T (Respiração-Temperatura), tendo sido detectados "platôs" entre as temperaturas de $20 \mathrm{e}$ 
$25^{\circ}, 25$ e $30^{\circ}, 30$ e $35^{\circ}$, bem como entre 20 e $30^{\circ}, 20$ e $35^{\circ}, 25$ e $35^{\circ}$ e entre 15 e $35^{\circ} \mathrm{C}$. Abaixo de $15^{\circ} \mathrm{C}$, ou seja, a $10^{\circ}$ e $5^{\circ} \mathrm{C}$ os diplópodos ficaram imobilizados pelo frio em situação de narcose reversível. No extremo superior, entre $35^{\circ}$ e $40^{\circ} \mathrm{C}$, foi detectado o menor índice de variação metabólica $\left(\mathrm{Q}_{10}=0,52\right)$. A $40^{\circ} \mathrm{C}$ os diplópodos apresentaram sinais de torpor pelo calor. Nos experimentos de ritmos de 24 horas as taxas respiratórias de Oxidus gracilis foram mais altas à noite que durante o dia, confirmando as observações largamente encontradas na literatura sobre os hábitos comportamentais noturnos dos diplópodos em geral. Tais hábitos sugerem uma maior economia energética e, consequentemente, maior controle da água perdida pela desidratação.

Palavras-chave: Diplópodos, temperatura, fotoperíodo, respiração, metabolismo.

\section{ABSTRACT}

The respiratory metabolism of Oxidus gracilis was searched considering the parameters temperature and photoperiod trying, in the first case, to detect accidental compensatory adjusts imposed by temperature variations, and in the second, the metabolic changes of the activity rhythm in the 24 hours period. In both cases the data were obtained in a microrespirometer of Warburg, using flasks of $20 \mathrm{ml}$ containing 0,3 $\mathrm{ml}$ of $14 \% \mathrm{KOH}$ solution for absorption of the $\mathrm{CO}_{2}$ exhaled in respiration. The tests with temperature were made at $5^{\circ}, 10^{\circ}, 15^{\circ}, 20^{\circ}, 25^{\circ}, 30^{\circ}, 35^{\circ}$ and $40^{\circ} \mathrm{C}$, and in those ones of metabolic rhythms, the measures were made only at $25^{\circ} \mathrm{C}$, during 24 hours, in which the data were collected during one hour every 3 hours. The data were shown as respiratory rates translated into $\mathrm{m} \mathrm{O}_{2} \mathrm{~g}(\text { live })^{-1}$.hour ${ }^{-1}$. The results showed the occurrence of one metabolic stablishing in the R-T curve (Respiration-Temperature), and "plateaux" were detected among the temperatures of $20^{\circ}$ and $25^{\circ}, 25^{\circ}$ and $30^{\circ}, 30^{\circ}$ and $35^{\circ}$, as well as among $20^{\circ}$ and $30^{\circ}, 20^{\circ}$ and $35^{\circ}$, and between $15^{\circ}$ and $30^{\circ} \mathrm{C}$. Below $15^{\circ} \mathrm{C}$, that is, at $10^{\circ}$ and $5^{\circ}$ the millipedes got emotionless by cold in a situation of reversible narcosis. At the high temperatures, between $35^{\circ}$ and $40^{\circ} \mathrm{C}$, it was detected the smallest index of metabolic range $\left(\mathrm{Q}_{10}=0,52\right)$, and at $40^{\circ} \mathrm{C}$ some millipedes showed the effects of torpor by the heat. In the tests of rhythms of 24 hours, the respiratory rates of Oxidus gracilis were higher at night than during the day, confirming data widely found in the literature about the nocturnal behavioral habits of millipedes in general. The results also suggest the existence of a metabolic control mechanism for temperature variations in Oxidus gracilis, and its nocturnal behavior could indicate, besides an expected energetic economy, a good resource to control the water loss by dehydration.

Key-words: millipedes, temperature, photoperiod, respiration, metabolism 
Os diplópodos são participantes ativos da cadeia energética dos ecossistemas terrestres, pois tal como as minhocas e cupins promovem a mistura de matéria orgânica e mineral do solo, que após ter transitado pelo intestino e expelido como fezes, facilita a ação dos decompositores menores, como fungos e bactérias, produtores de ácidos húmicos. Diplópodos seriam, assim, importantes indicadores da fertilidade do solo (BYZOVA, 1967; CRAWFORD， 1976; DANGERFIELD \& TELFORD, 1989; HOPKIN \& READ, 1992).

Diante desta vital importância ecológica é fundamental que certos aspectos básicos da fisioecologia dos diplópodos sejam estudados, destacando-se o da determinação do seu metabolismo respiratório, que além de fornecer subsídios para a compreensão do grau de participação desses animais na "Energy Turnover" do solo (PHILLIPSON, 1966; REICHLE, 1968; MacNEILL \& LAETON, 1970; CRAWFORD, 1979), ajudam a compreender o grau de dependência metabólica dos diplópodos em relação aos fatores ambientais, entre os quais a temperatura e o fotoperíodo.

O metabolismo respiratório, tal como outros processos fisiológicos é influenciado pela temperatura de acordo com o princípio de Van't Hoff-Arrehnius e isto também ocorre nos animais poiquilotermos. Esta influência em geral permite um ajustamento à curva R-T (Respiração-Temperatura), embora Bullock (1955), Prosser (1955) e Fry (1958) tenham enfatizado a possibilidade de poiquilotermos apresentarem ajustes compensatórios de vários tipos e seres mais ou menos independentes das variações de temperatura. Tais ajustes permitem revelar "platôs" na curva R-T, onde baixos valores de $\mathrm{Q}_{10}$ (coeficiente de temperatura) podem ser interpretados como um sintoma de compensação metabólica, isto é, maior economia energética (WIESER, 1973).

Em diplópodos, pesquisas envolvendo relações metabólicas com a temperatura têm sido efetuadas com espécies aclimatadas ou nativas de regiões temperadas e tropicias. Na Polônia destacam-se os trabalhos de Gromysz-Kalkowska \& Stojalowska (1966) e de Gromysz-Kalkowska (1970, 1973, 1974, 1976a, 1976b e 1979). Na Grécia o assunto foi abordado por Stamou \& Iatrou (1993). Na Índia podem ser citados os trabalhos de Dwarakanath (1971a, 1971b) e de Dwarakanath et al. $(1972,1973)$. Nos Estados Unidos a questão foi abordada por Stewart \& Woodring (1973) e, no Brasil, trabalhos já foram realizados por Penteado \& Mendes (1981), Wernick et al (1983), Boccardo \& Penteado (1995a, b) e Boccardo \& Penteado (1998).

Os ritmos de atividade são amplamente distribuídos na natureza e provavelmente são regulados por um "relógio" fisiológico interno, que obedece a padrões 
fixados geneticamente; muitas vezes envolvendo níveis de independência das variações físicas do ambiente, tais como a temperatura, a umidade, o fotoperíodo, as estações do ano, as fases lunares e, até mesmo as marés, no caso dos animais marinhos. Destes fatores citados, a luz natural é, porém, reconhecidamente, o mais importante "Zeitgeber" dos ritmos circadianos (ASCHOFF, 1963).

Pesquisas envolvendo os ritmos de atividade em diplópodos encontram-se embasados em trabalhos apresentados por diversos autores, entre os quais Park et al (1931), Cloudsley-Thompson (1951), Stojalowska (1960), Paulpandian (1965), Toye (1966c), Brandon (1967), Stewart (1969), Mead \& Gilhodes (1974), Gilhodes (1974), Wooten et al. (1975), Bano \& Krishnamoorty (1979) E Baker (1988). No Brasil o assunto já foi abordado por Penteado \& Mendes (1983), Pedro \& Penteado (1986), Levy Jr. (1993) Levy Jr. \& Penteado (1995) e Boccardo \& Penteado (1995b).

Neste trabalho o metabolismo respiratório do diplópodo Oxidus gracilis foi relacionado com os parâmetros temperatura e fotoperíodo, visando detectar eventuais ajustes compensatórios do metabolismo no que se refere à variações da temperatura, bem como as alterações metabólicas envolvidas no ritmo de 24 horas.

\section{MATERIAL A MÉTODOS}

Oxidus gracilis (Koch, 1847) é um diplópodo estrongilosomídeo, também referido na literatura como pertencente à família Paradoxosomatidae. O gênero é de origem asiática (Japão, Coréia, China e ilhas Riu-Kiu). Quanto à espécie, ela é considerada cosmopolita, (HOFFMAN, 1979) ou sub-cosmopolita (GOLOVATCH, 1990), com ampla distribuição no continente americano (SCHUBART, 1944). É facilmente espalhada pelo homem, já que apresenta um caráter sinantrópico, vivendo em quintais, jardins, estufas e pomares. É mais rara, porém, nos biótopos virgens (SCHUBART, 1953). É uma espécie de pequeno tamanho, adultos atingindo cerca de 4 cm (SCHUBART, 1953). A espécie é comumente encontrada durante o ano inteiro, escasseando apenas nos meses sem chuva e de temperatura muito baixas. No Brasil a ocorrência de indivíduos jovens é maior, da metade da primavera até o final do verão.

Os exemplares de Oxidus gracilis utilizados neste trabalho foram coletados no jardim experimental da UNESP - Campus de Rio Claro - SP (22 $24^{\prime}$ ' $36^{\prime}$ 'S; $47^{\circ}$ 33'36'W), em lugares úmidos, protegidos pelo "litter"; à sombra das árvores. Nestes ambientes onde eles vivem, as temperaturas situam-se em torno de $18^{\circ} \mathrm{C}$ ao longo do ano (SILVA, 1997).

No laboratório, os diplópodos foram mantidos em câmara climática (temperatura entre $20^{\circ}$ e $25^{\circ} \mathrm{C}$ ), em terrários providos de folhas, cascas de madeira apodrecida, xaxim e terra umidecida trazida dos locais de coleta. 
O metabolismo respiratório foi determinado em respirômetro de Warburg, segundo o método usual proposto por Dixon (1943). Foram utilizados frascos de aproximadamente $20 \mathrm{ml}$ de capacidade, aos quais foram adicionados $0,3 \mathrm{ml}$ de solução de $\mathrm{KOH}$ a $14 \%$ para a absorção de $\mathrm{CO}_{2}$ expirado. Cada série experimental envolveu 7 conjuntos de manômetro/frasco; 6 deles contendo 1 diplópodo por frasco e o sétimo servindo como termobarômetro. Nos experimentos foram utilizados diplópodos machos e fêmeas, jejunos de 24 horas, em fase de intermuda e de inatividade sexual. $\mathrm{O}$ procedimento adotado foi o do metabolismo de rotina, onde os animais não sofrem contenção dos movimentos dentro dos frascos.

Nos experimentos referentes às relações com a temperatura, diferentes lotes de animais foram submetidos a testes com temperaturas de $5^{\circ}, 10^{\circ}, 15^{\circ}, 20^{\circ}, 25^{\circ}, 30^{\circ}$, $35^{\circ}$ e $40^{\circ} \mathrm{C}$. Nos experimentos de ritmos metabólicos a temperatura foi fixada em $25^{\circ} \mathrm{C}$ e medidas respirométricas de 1 hora foram tomadas de $3 \mathrm{em} 3$ horas, em fotoperíodo normal (dia, claro/noite escuro), durante 24 horas, iniciando-se às 18 horas de um dia e terminando às 18 horas do dia seguinte. As taxas respiratórias $\left(\mathrm{vO}_{2}\right)$ foram expressas em

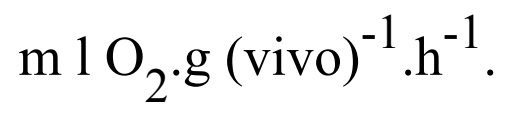

Os resultados obtidos foram analisados segundo Dixon e Massey Jr. (1957), Zar (1974) e Sokal e Rohlf (1981).

\section{RESULTADOS E DISCUSSÃO}

\section{Temperatura}

$\mathrm{Na}$ natureza, com pequenas exceções, as faixas térmicas nas quais os processos vitais ocorrem situam-se entre 0 e $40^{\circ} \mathrm{C}$, mas a maioria dos animais distribui-se apenas dentro de limites bastante estreitos, aos quais acham-se ajustados. Desse modo a temperatura limita a distribuição dos animais e ao mesmo tempo determina as suas atividades (PROSSER, 1973).

As respostas de Oxidus gracilis a variações de temperatura, inferidas das taxas respiratórias medidas a $5^{\circ}, 10^{\circ}, 15^{\circ}, 20^{\circ}, 25^{\circ}, 30^{\circ}, 35^{\circ}$ e $40^{\circ} \mathrm{C}$ foram obtidas de um total de 157 indivíduos, de massa corpórea variando de 0,035 a 0,090 g. Os resultados constam da Tabela 1 e da Figura 1. A Figura 2 retrata as alterações metabólicas expressas em termos de $\mathrm{Q}_{10}$ calculados de acordo com a equação: 


$$
Q_{10}=\left(\frac{K_{1}}{K_{2}}\right)^{\frac{10}{t_{1}-t_{2}}}
$$

de Schmidt-Nielsen (1990) e Prosser (1991), onde, $K_{1}$ e $K_{2}$ são as taxas respiratórias comparadas duas a duas, de $5^{\circ} \mathrm{em} 5^{\circ} \mathrm{C}$, na seqüência de experimentação e $\mathbf{t}_{\mathbf{1}}$ e $\mathbf{t}_{\mathbf{2}}$, as respectivas temperaturas envolvidas na referida seqüência.

Os dados da curva geral de Respiração-Temperatura também foram estudados estatisticamente usando-se a análise de variância One-way - Anova. O teste acusou um valor de $\mathrm{F}=38,41$, que comparado com o $\mathrm{F}$ crítico a nível de $5 \%(2,09)$, indicou alta significância na relação.

Empregando-se a seguir sobre os dados da Tabela 1 e Figura 1 o teste $a$ posteriori (Newman Keuls multiple-range test) para amostras com $\mathrm{n}$ desiguais, os resultados indicaram platôs metabólicos entre $15^{\circ}$ e $35^{\circ} \mathrm{C}, 20^{\circ}$ e $25^{\circ} \mathrm{C}, 20^{\circ}$ e $30^{\circ} \mathrm{C}, 20^{\circ}$ e $35^{\circ} \mathrm{C}, 25^{\circ}$ e $30^{\circ} \mathrm{C}, 25^{\circ}$ e $35^{\circ} \mathrm{C}$ e $30^{\circ}$ e $35^{\circ} \mathrm{C}$. Em termos de valores de $\mathrm{Q}_{10}$, os maiores incrementos térmicos ocorreram entre $5^{\circ}$ e $10^{\circ} \mathrm{C}$ e $10^{\circ}$ e $15^{\circ} \mathrm{C}$. No extremo superior, entre $35^{\circ}$ e $40^{\circ} \mathrm{C}$ detectou-se o menor valor de $\mathrm{Q}_{10}(0,52)$ e entre $15^{\circ}$ e $35^{\circ} \mathrm{C}$ os $\mathrm{Q}_{10}$ aproximaram-se da unidade (Tabela 1 e Figura 2).

Tabela 1 . Taxas respiratórias $\left(\dot{\mathrm{vO}}_{2}=\mu 1 \mathrm{O}_{2} \cdot \mathrm{g}^{-1} \cdot \mathrm{h}^{-1}\right)$ de Oxidus gracilis obtidas a $5^{\circ}$, $10^{\circ}, 15^{\circ}, 20^{\circ}, 25^{\circ}, 30^{\circ}, 35^{\circ}$ e $40^{\circ} \mathrm{C}$, sem distinção de sexos. Massa corpórea entre 0,035 e $0,090 \mathrm{~g}$. Valores de Q10 foram calculados para cada intervalo de $5^{\circ} \mathrm{C}$.

\begin{tabular}{||c||c||c||c||c||}
\hline $\begin{array}{c}\text { Temperatura } \\
\left({ }^{\circ} \mathrm{C}\right)\end{array}$ & $\mathrm{n}$ & $\begin{array}{c}\text { Massa } \\
\text { Corpórea }\end{array}$ & $\dot{\mathrm{vO}}_{2}$ & $\mathrm{Q}_{10}$ \\
& & $\begin{array}{c}\text { média } \pm \\
\mathrm{dp}\end{array}$ & & \\
\hline \hline & & & & \\
\hline \hline 05 & 20 & $\begin{array}{c}0,0680 \pm \\
0,0086\end{array}$ & $\begin{array}{c}169,1282 \pm \\
69,1714\end{array}$ & \\
\hline \hline 10 & 20 & $\begin{array}{c}0,0603 \pm \\
0,0104\end{array}$ & $\begin{array}{c}258,2718 \pm \\
66,7690\end{array}$ & 2,33 \\
\hline \hline 15 & 21 & $0,0609 \pm$ & $356,2243 \pm$ & 1,90 \\
& & & & \\
\hline \hline
\end{tabular}




\begin{tabular}{||c||c||c||c||c||} 
& & 0,0087 & 51,7843 & \\
\hline \hline 20 & 22 & $\begin{array}{c}0,0637 \pm \\
0,0132\end{array}$ & $\begin{array}{c}407,1305 \pm \\
101,9299\end{array}$ & 1,30 \\
& 18 & $\begin{array}{c}0,0635 \pm \\
0,0122\end{array}$ & $\begin{array}{c}464,2253 \pm \\
82,7419\end{array}$ & 1,30 \\
\hline \hline 25 & 17 & $\begin{array}{c}0,0623 \pm \\
0,0124\end{array}$ & $\begin{array}{c}446,9093 \pm \\
80,8051\end{array}$ & 0,92 \\
\hline \hline 30 & 21 & $\begin{array}{c}0,0649 \pm \\
0,0107\end{array}$ & $\begin{array}{c}428,3711 \pm \\
131,9593\end{array}$ & 0,92 \\
\hline \hline 35 & 19 & $\begin{array}{c}0,0613 \pm \\
0,0136\end{array}$ & $\begin{array}{c}308,5855 \pm \\
114,6643\end{array}$ & 0,52 \\
\hline \hline 40 & 19 & & \\
\hline \hline
\end{tabular}

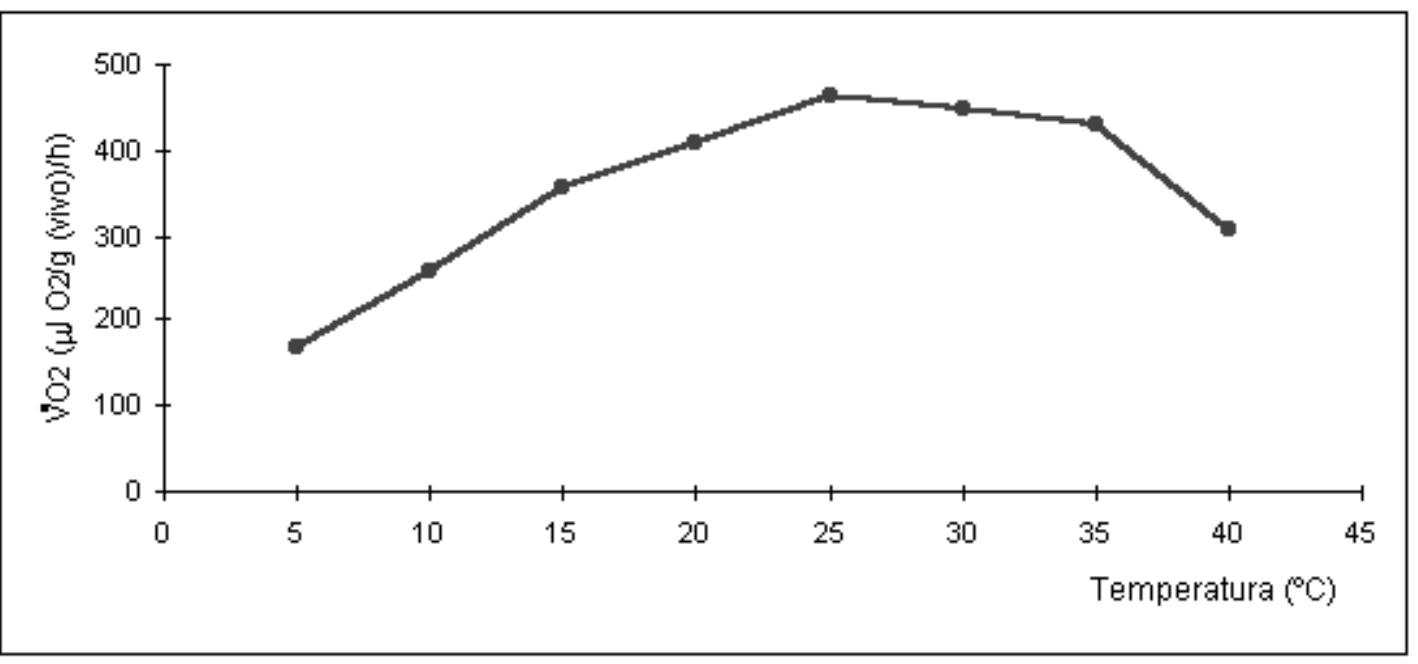

Figura 1. Taxas respiratórias de Oxidus gracilis em oitos diferentes temperaturas (5 a $40^{\circ} \mathrm{C}$ ), sem distinção de sexos. Massa corpórea entre 0,035 e 0,090 g.

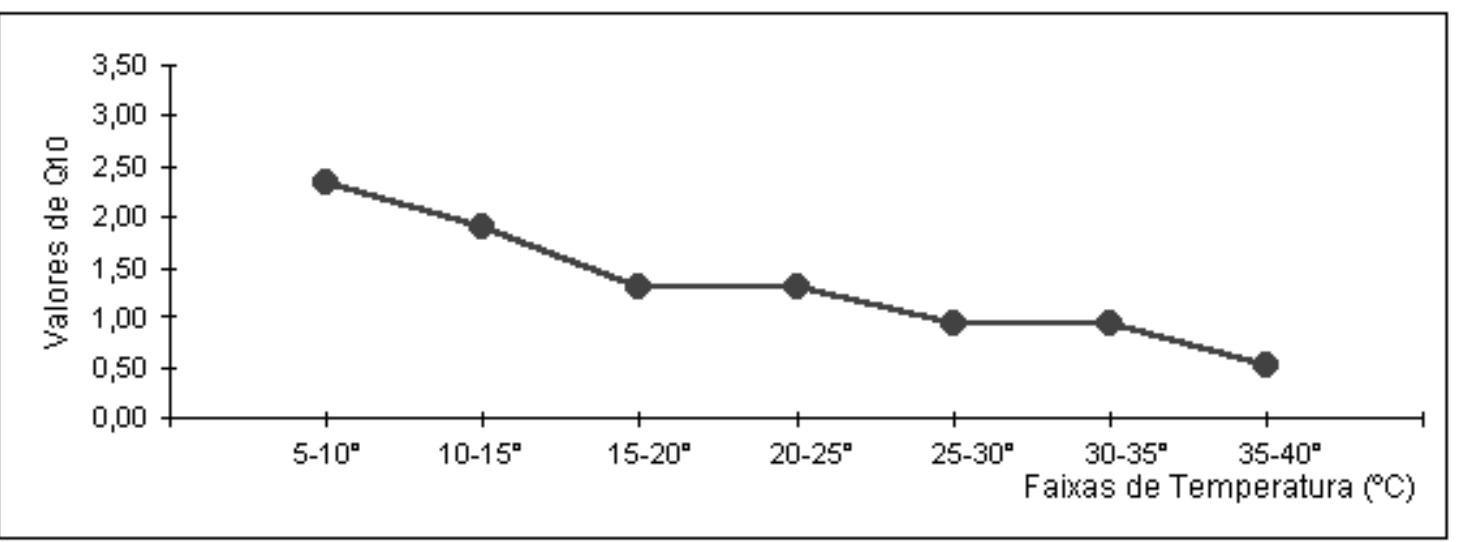


Figura 2. Valores de $\mathrm{Q}_{10}$ de Oxidus gracilis em intervalos de $5^{\circ} \mathrm{C}$, a partir das taxas respiratórias obtidas nas temperaturas de $5^{\circ}, 10^{\circ}, 15^{\circ}, 20^{\circ}, 25^{\circ}, 30^{\circ}, 35^{\circ}$ e $40^{\circ} \mathrm{C}$.

A análise dos dados envolvendo as relações metabólicas de Oxidus gracilis com a temperatura sugere a possibilidade de um controle do metabolismo relativamente eficiente entre $15^{\circ}$ e $35^{\circ} \mathrm{C}$ (Figura 1). Esta observação é reforçada quando é analisada em termos de $\mathrm{Q}_{10}$ (Figura 2), índice, que de um modo geral representa a capacidade adaptativa dos animais, ou seja, onde baixos valores indicam economia energética (WIESER, 1973), ou compensação metabólica. Desta análise devem ser descartados os valores obtidos no extremo superior (entre $35^{\circ}$ e $40^{\circ} \mathrm{C}$ ), onde os diplópodos ficaram expostos aos efeitos do calor excessivo (torpor pelo calor) e os do extremo inferior, cujos altos índices de $\mathrm{Q}_{10}(2,33$ e 1,90$)$, poderiam indicar incapacidade de ajustamento, devido aos efeitos da narcose pelo frio nas temperaturas de $5^{\circ}$ e $10^{\circ} \mathrm{C}$.

Dados da literatura sobre diplópodos atestam que Gromysz-Kalkowska \& Stojalowska (1966) obtiveram um platô entre 22 e $25^{\circ} \mathrm{C}$ empregando a mesma espécie O. gracilis, porém adaptada em estufas de plantas na Polônia, e que também foi caracterizado por um baixo valor de $\mathrm{Q}_{10}$ próximo à unidade. Em outras duas espécies, Strongylosoma pallipes (GROMYSZ-KALKOWSKA, 1973) e Polydesmus complanatus (GROMYSZ-KALKOWSKA, 1970) estreitamente aparentadas com $\boldsymbol{O}$. gracilis, foram encontrados dois platôs (entre $10-13^{\circ} \mathrm{C}$ e $22-25^{\circ} \mathrm{C}$ ) em $S$. pallipes aclimatados a $21^{\circ} \mathrm{C}$, mas nenhum platô em $P$. complanatus, embora tenham sido encontrados nesta mesma espécie, baixos valores de $\mathrm{Q}_{10}$, entre 13 e $16^{\circ} \mathrm{C}, 19$ e $22^{\circ} \mathrm{C}$ e 22 e $25^{\circ} \mathrm{C}$ (GROMYSZ-KALKOWSKA, 1970). Nestes estudos, a autora considerou estas gamas térmicas como as temperaturas ótimas fisiológicas dessas espécies, já que estariam relacionadas com manifestações de compensação metabólica devido a mecanismos homeostáticos, tanto de natureza endógena, como exógena. A mesma autora (GROMYSZ-KALKOWSKA, 1974) estudando Glomeris connexa em diferentes estações do ano e com variada massa corpórea, encontrou platôs em posições aleatórias na curva R-T. Para a autora, os resultados obtidos com Glomeris connexa indicariam que o consumo de oxigênio desta espécie é mais independente da temperatura ambiental, do que o de $P$. complanatus, esta considerada a espécie mais dependente. Num outro diplópodo, Polyzonium germanicum, Gromysz-Kalkowska (1976b) encontrou diversos valores de $\mathrm{Q}_{10}$ menores que 1,5 espalhados de maneira aleatória nas curvas $\mathrm{R}-\mathrm{T}$ e sem nenhuma relação com as estações do ano. Hall $\boldsymbol{e t}$ al. (1969) registraram dois diferentes platôs para dois diplópodos cianogênicos, entre $20-25^{\circ} \mathrm{C}$ para Pleuroloma flavipes bulteri e entre $15-20^{\circ} \mathrm{C}$ para Euryurus leachii. Estes autores afirmaram que tais platôs poderiam ser referidos às suas temperaturas de preferência ou aos intervalos da temperatura ótima fisiológica das espécies. Para outros pesquisadores (EDWARDS \& PÉREZGONZALES, 1954), baixos índices de $\mathrm{Q}_{10}$, realmente, podem ser considerados como de 
valor adaptativo e, tal como reforçado por Wieser (1973), eles garantiriam aos animais uma maior economia energética, capacitando-os a suportarem melhor as mudanças de temperatura. Rao \& Bullock (1954) sugeriam, além disso, que os valores de $\mathrm{Q}_{10}$, medidos em função de várias atividades dos organismos sofreriam influências, não só da temperatura, como também do habitat e do tamanho corpóreo dos animais. Wernick et al. (1983) estudando os diplópodos polidesmídeos Leptodesmus dentellus e Sandalodesmus gasparae, notaram a influência do tamanho nos valores de $\mathrm{Q}_{10}$ ao verificarem que $S$. gasparae, a espécie menor, apresentava os menores índices de $\mathrm{Q}_{10} \mathrm{em}$ toda uma gama de variação de temperaturas, quando comparadas com $L$. dentellus, a espécie de tamanho maior. Relações diretas dos valores de $\mathrm{Q}_{10}$ com o tamanho também foram notadas por Dwarakanath (1971b) no diplópodo Spirostreptus asthenes, porém, Wooten \& Crawford (1974) descreveram uma relação inversa em Orthoporus ornatus, espécies dos desertos norte-americanos.

Analisando a influência do habitat nos valores de $\mathrm{Q}_{10}$, Stewart \& Woodring (1973), em seus estudos com os diplópodos americanos Pachydesmus crassicutis e Orthoporus texicolens, bem como Wooten \& Crawford (1974) com Orthorporus ornatus, verificaram que os menores valores de $\mathrm{Q}_{10}$ pertenciam à espécie de ambiente mais úmido (P. crassicutis). Em L. dentellus e $S$. gasparae, os platôs encontrados por Wernick et al (1983), possivelmente retrataram o modo de vida desses diplópodos. Assim, L. dentellus, geralmente encontrados em troncos apodrecidos, em lugares úmidos e sombrios, mostrou um platô entre 15 e $20^{\circ} \mathrm{C}\left(\mathrm{Q}_{10}=1,39\right)$. S. gasparae, que freqüentemente se expõe a temperaturas mais elevadas, deslocando-se sobre o "litter" muitas vezes até em lugares desprotegidos de sombra, apresentou platô entre 20 e $25^{\circ} \mathrm{C}$ $\left(\mathrm{Q}_{10}=1,07\right)$. Boccardo \& Penteado (1995a) encontraram em Gymnostreptus olivaceus valores relativamente baixos entre 10 e $35^{\circ} \mathrm{C}$, o que, segundo os autores, retratariam uma capacidade de adaptação mais ampla desse diplópodo às variações de temperatura. Este fato foi concordante com os menores valores de $\mathrm{Q}_{10}$ obtidos para Oxidus gracilis, aqui encontrados entre 15 e $35^{\circ} \mathrm{C}$. Em Glomeris balcanica, Stamou \& Iatrou (1993) enfatizaram que os baixos valores de $\mathrm{Q}_{10}$ encontrados dentro da faixa térmica de 5 a $27^{\circ}$ $\mathrm{C}$ denotaria a existência de um mecanismo compensatório adaptativo, garantindo custos de manutenção bem distribuídos e atividade balanceada dentro de uma ampla faixa de temperatura, ou seja, a baixa eficiência de transformação energética da espécie seria compensada por uma atividade contínua, fazendo com que G. balcanica possa atuar como um componente funcional dominante do subsistema do solo do ambiente estudado.

Penteado \& Mendes (1981), quando estudaram o metabolismo respiratório do diplópodo Rhinocricus padbergi, não levaram em conta o problema do habitat nos 
valores de $\mathrm{Q}_{10}$. entretanto, comparando-se as taxas metabólicas e índices de $\mathrm{Q}_{10}$ de $R$. padbergi (exemplares de massa corpórea entre 1,90 e 2,50 g), com as taxas e índices de G. olivaceus (exemplares entre 2,0 e 3,0g), obidos por Boccardo \& Penteado (1995a) observa-se que os valores registrados para $R$. padbergi foram, quase sempre, superiores aos de $G$. olivaceus, demonstrando menor taxa de adaptação de $R$. padbergi, considerando-se os mesmos intervalos de temperatura.

\section{Ritmo de 24 horas.}

Nos animais, as relações que a ritmicidade mantém com a taxa metabólica, têm sido objeto de numerosas investigações, pois os ritmos diurno e noturno, apesar de coincidirem com as alterações do nível metabólico (JANDA \& MRCIAK, 1943 apud CLOUDSLEY-THOMPSON, 1961), podem, ou não, persistirem em condições de laboratório, dependendo de serem, mais acentuadamente "endógenos" ou "exógenos". Diplópodos, assim como quilópodos, são animais geralmente ativos em condições de alta umidade, mas sua locomoção se restringe quase sempre aos períodos noturnos, já que permanecem geralmente abrigados e escondidos durante o dia. Levando em conta que esses artrópodos são relativamente desprotegidos contra a perda de água por evaporação (PENTEADO \& MENDES, 1982), é de se esperar que a sua atividade noturna seja favorecida pelas condições de menor temperatura e maior umidade relativa ambiental.

As taxas respiratórias de Oxidus gracilis registradas nos experimentos de ritmos metabólicos obtidos em condições de fotoperíodo normal (dia claro/noite escuro) foram determinadas em diplópodos pesando entre 0,042 e 0,089 g e encontram-se na Figura 3. A Tabela 2 apresenta as médias dos dados originais de cujos valores foi construída a figura citada. A curva da Figura 3 revela que foram mais altas as taxas respiratórias noturnas que as diurnas. No período entre a 0 e 6 horas, ou seja, durante a madrugada, registraram-se as maiores taxas do experimento. Após as 6 horas as taxas caíram acentuadamente até o meio dia e depois mais lentamente até o final do experimento.

Tabela 2 - Taxas respiratórias médias $\left(\mathrm{V}_{2}=\mu \mathrm{IO}_{2} \cdot \mathrm{g}^{-1} \cdot \mathrm{h}^{-1}\right)$, diurnas e noturnas de Oxidus gracilis sob a condição de fotoperíodo normal (dia claro/noite escuro). Massa corpórea entre 0,042 e 0,089 g, Machos e fêmeas indistintamente (18 animais ao todo; 6 em cada série). 
FOTOPERÍODO NORMAL

\begin{tabular}{|c|c|c|c|c|c|c|c|c|c|}
\hline Horas & 18 & 21 & 0 & 3 & 6 & 9 & 12 & 15 & 18 \\
\hline VO2 & 280,49 & 356,30 & 475,26 & 508,02 & 461,08 & 310,63 & 202,47 & 156,95 & 135,20 \\
\hline SD & $(24,93)$ & $(82,65)$ & $(108,05)$ & $(38,11)$ & $(48,41)$ & $(96,52)$ & $(53,85)$ & $(31,40)$ & $(16,32)$ \\
\hline
\end{tabular}

SD = desvio-padrão

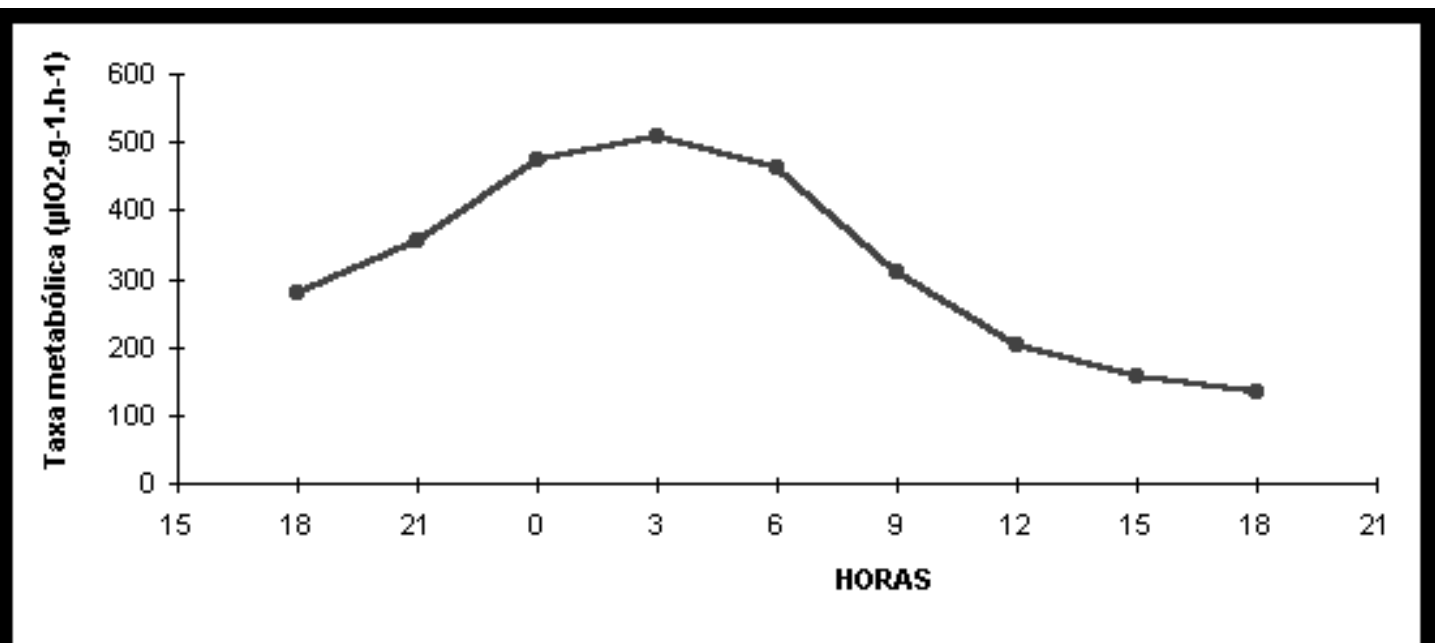

Figura 3. Taxas respiratórias diurnas e noturnas de Oxidus gracilis sob a condição de fotoperíodo normal (dia claro/noite escura), a $25^{\circ} \mathrm{C}$. Os dados numéricos constam da Tabela 2.

Em estudos sobre ritmos de atividade em diplópodos Park et al. (1931), detectaram em Polydesmus serratus maior atividade ao redor da meia noite, em consonância com o aumento da umidade relativa e a diminuição da temperatura ambiental. Em Spirobolus marginatus, Park (1935) descreveu um ritmo noturno persistente, mesmo em escuridão contínua e déficits de saturação inalterados. CloudsleyThompson (1951) também observou esse fenômeno nas espécies africanas Ophistreptus sp e Oxydesmus platycercus, mas não em duas espécies aclimatadas em estufas na Inglaterra, Paradesmus gracilis e Blaniulus guttulatus, que foram mais sensíveis às mudanças do fotoperíodo. Já Paulpandian (1965), verificou redução da atividade em 
escuridão contínua, na espécie indiana Arthrosphaera dalyi, com uma maior regularidade do ritmo sob luz permanente. Todavia, trabalhando em condições constantes de temperatura, umidade e iluminação, Brandon (1967) não notou diferenças nos picos de maior atividade de duas espécies, Oxidus gracilis e Pachydesmus crassicutis, que se mantinham ao redor das 22 horas. Essa regularidade, porém, não se manteve quando $P$. crassicutis foi submetido a diferentes padrões de fotoperíodo e rastreado por meio de detectores ultra-sônicos, conforme relatado por Stewart (1969). Baker (1988) também descreveu um ritmo noturno na espécie de diplópodo, Ommatoiulus moreletii (originário de Portugal; introduzido na Austrália), mas neste caso os padrões rítmicos foram alterados pelas estações do ano.

Aumentos de atividade locomotora e alimentar nos horários de nascer e do pôr-do-sol, foram detectados em diplópodos por Wooten et al. (1975) na espécie desertícola Orthoporus ornatus e interpretados como de origem exógena. Uma acentuada influência do fotoperíodo foi igualmente registrada por Bano \& Krishnamoorty (1979), na espécie indiana Jonespeltis splendidus, na qual a escuridão contínua alterou, drasticamente, o ritmo circadiano do animal.

O fotoperíodo natural, via de regra, não interfere no ritmo circadiano de animais cavernícolas. Ainda, assim, Mead \& Gilhodes (1974), estudando o comportamento do diplópodo cavernícola, Blaniulus lichtensteini, verificaram a persistência de um ritmo de atividade do animal, que certamente dependia de um relógio interno, que agia mesmo nas condições de escuridão permanente. Num outro diplópodo cavernícola, Callipus foetidissimus, Gilhodes (1974), também encontrou um ritmo regular, mas independente do fotoperíodo natural, de 24 horas, que o autor interpretou como sendo endógeno.

Boccardo \& Penteado (1995b) relataram que o diplópodo Gymnostreptus olivaceus é caracterizada por uma atividade noturna sobre condições de fotoperíodo normal, mas, quando o fotoperíodo foi invertido, reduziu-se a atividade noturna em relação ao fotoperíodo normal, mas não o ritmo totalmente. Os autores admitiram haver uma persistência do ritmo endógeno onde a luz não foi considerada um importante sincronizador da atividade da espécie, já que foi insuficiente para bloquear o despertar do relógio biológico, mantendo-se, assim, a tendência natural das atividades noturnas, principalmente quando os testes foram feitos em condições de iluminação e escuridão contínuas. Este fato também foi observada por Levy Jr. (1993) em Pseudonannolene tricolor, no qual a dependência da luz foi, igualmente, baixa .

A atividade rítmica, quando examinada sob o enfoque respirométrico, pode apresentar, em alguns casos, sincronismo com picos de atividade locomotora ou fisiológica em diplópodos. Este fato ocorreu com as espécies, Arthrosphaera dalyi, estudada por Paulpandian (1966) e Spirostreptus asthenes, estudada por Dwarakanatha \& Job (1966), nas quais o aumento da respiração, à noite, coincidiu com o período em 
que os animais são mais ativos. Nos diplópodos Glomeris connexa e Polyzonium germanicum (GROMYSZ-KALKOWSKA, 1974; 1976b), mudanças no ritmo respiratório não foram significativas após um período de 24 horas, mas em Rhinocricus padbergi (PENTEADO \& MENDES, 1983) foi registrado um efeito do fotoperíodo sobre o metabolismo. Em contraste, em Pseudonannolene tricolor (LEVY Jr., 1993), a inversão do fotoperíodo não interferiu com o padrão de atividade noturna do diplópodo, sendo que as taxas respiratórias noturnas foram superiores às diurnas. Já em Gymnostreptus olivaceus (BOCCARDO \& PENTEADO, 1995b), quando submetido a fotoperíodo normal, as taxas respiratórias confirmaram a periodicidade noturna revelada pelos experimentos de atividade locomotora e, mesmo quando expostos à inversão do fotoperíodo, escuridão contínua e iluminação contínua, não ocorreram alterações do padrão rítmico (taxas respiratórias usualmente maiores à noite do que de dia).

Infelizmente, devido ao seu pequeno tamanho e baixa massa corpórea, Oxidus gracilis não se mostrou ser uma espécie apropriada para a actografia convencional, nos moldes do que feito com $R$. padbergi e G. olivaceus. Em experimentos de respirometria, porém, conforme verificado em fotoperíodo normal, a espécie comportou-se dentro do padrão típico registrado para outros diplópodos, constatando-se que as maiores taxas respiratórias, ocorreram no período noturno, quando geralmente são menores os riscos de desidratação.

\section{AGRADECIMENTOS}

Os autores agradecem ao Departamento de Zoologia do Instituto de Biociências da UNESP, Campus de Rio Claro, em cujos laboratórios de fisiologia os experimentos foram realizados e à CAPES, que concedeu bolsa de pós-graduação possibilitando ao primeiro autor a obtenção do Mestrado em Zoologia.

\section{REFERÊNCIAS}

ASCHOFF, J. Comparative physiology: diurnal rhytms. Ann. Rev. Physiol. V.25, p.581600,1963

BAKER, G. H. Patterns in the activity of the millipede Ommatoiulus moreleti (Diplopoda, Julidae). Aust. ent. Mag., v.15, n.4, p.127-139, 1988

BANO, K.; KRISHNAMOORTY, R.V. Circadian rhytms in the sociability and locomotor activities of the millipede, Jonespeltis splendidus Verhoeff. Behav. Neural Biology, v.25, p.573-582, 1979 
BOCCARDO, L; PENTEADO, C.H.S. Preferências Térmicas e respostas metabólicas em relação à temperatura e ao tamanho em Gymnostreptus olivaceus Schubart, 1944 (Diplopoda, Spirostreptida). Rev. Bras. Biol., v.3, n.55, 445-56, 1995a.

BOCCARDO, L. PENTEADO, C.H.S. Locomotor and metabolic activities of Gymnostreptus olivaceus (Diplopoda, Spirostreptida) at different photoperiod conditions. Comp. Biochem. Physiol. V.112a, p.611-617, $1995 \mathrm{~b}$.

BOCCARDO, L. \& PENTEADO, C.H.S. Thermal limits in relation to acclimation in the neotropical millipede Gymnostreptus olivaceus (Diplopoda, Spirostreptidae). Stud. Neotrop. Fauna \& Environm. v. 33, p. 165-169, 1998.

BRANDON, S. Disruption of the circadian rhythm of two by low-level gama radiation. Proc. L. Acad. Sc. v.30, p. 70-73,1967

BULLOCK, T.H. Compensation for temperature in the metabolis and activity of poikilotherms. Biol. Rev. v. 30, p.311-342, 1955,

BYZOVA, J.B. Respiratory metabolism in some millipedes (Diplopoda). Rev. Ecol. Biol. Sol. v.4, n.4, p.611 - 624, 1967.

CLOUDSLEY-THOMPSON, J.L. Studies in diurnal rhythms. I. Rhythmic behavior in millipedes. J. Exp. Biol., v.28, n.2, p.165-172, 1951.

CLOUDSLEY-THOMPSON, J.L. Rhythmic activity In: Animal Physiology and Behavior. New York \& London: Academic Press, 1961, 236p.

CRAWFORD, C.S. Feeding-season production in the desert millipede Orthophorus ornatus (Girard) (Diplopoda). Oecologia, v.24, p.265-276, 1976.

CRAWFORD, C.S. Assimilation, respiration and production: (a) Invertebrates. In: CRAWFORD, C.S. Arid-land Ecosystems: Structure, Functioning and Megagement. vol. I. Cambridge University Press, 1979. p.717-729.

DANGERFIELD, J.M., TELFORD, S.R. Are millipedes important for soil fertility? Zimbabwe Science News, v.23, n.7-9, p.66-68, 1989.

DIXON, M. Manometric Methods. Cambridge: Univ. Press \& New York: MacMillan, 1943. $x i v+155 p$.

DIXON, W.J. \& MASSEY Jr., F.J. Introduction to statistical analysis. Nova York, Mac Graw Hill, 488 p., 1957.

DWARAKANATH, S.K. The influence of body size and temperature upon the oxygen consumption in the millipede Spirostreptus asthenes (PococK). Comp. Biochem. 
Physiol. v.38A, p.351-358, 1971a.

DWARAKANATH, S.K. Effect of temperature on the oxygen consumption in a tropical millipede Harpurostreptus sp. Proc. Indian Acad. Sci. (Sec. B), v.73, n.1, p.4-7, $1971 b$.

DWARAKANATH, S.K., JOB, S.V. Studies on transpiration in millipedes. II. Respiration and water loss in Spirostreptus asthenes. Proc. Indian Acad. Sci., v.62, p.224-228, 1966.

DWARAKANATH, S.K. BERLIN, O.G.W., PANDIAN, R.S. Respiratory metabolism in Polydesmus gracilis (Diplopoda) as a function of temperature and sex. Curr. Sci., v.41, n.5, p.184-185, 1972.

DWARAKANATH, S.K., BERLIN, O.G.W., PANDIAN, R.S. Oxygen consumption as a function of size and temperature in pill millipede Arthrosphaera disticta (Pocock). Monit. Zool. Ital., v.7, p.43-50, 1973.

EDWARDS, G.A.; PÉREZ-GONZALES, M.D. The influence of temperature upon the respiratory metabolism of certain aquatic insects. Acta Physiol. Latinoam. V.4, n.3, p.121-132, 1954

FRY, F.E.J. Temperature compensation. Ann. Rev. Physiol. v.20, p.207-224, 1958.

GILHODES, J.C. Étude du rhythme d'activité locomotrice de Callipus foetidissimus Bröl (Diplopode) en libre cours. Rev. Comp. Animal., v.8, p.63-70, 1974.

GOLOVATCH, S.I. Diplopoda from the Nepal Hymalayas. The first Paradoxosomatidae. Spixiana v.13, n.1, p.9-24, 1990.

GROMYSZ-KALKOWSKA, K. The influence of body weight, external temperature, seasons of the year and fasting on respiratory metabolism in Polydesmus complanatus L. (Diplopoda). Folia Biol., v.18, n.4, p.311-326, 1970.

GROMYSZ-KALKOWSKA, K. Some exogenus and endogenus effects on rate of respiration in Strongylosoma pallipes (Olivier) (Diplopoda) and behavior in temperature gradient. Folia Biol., v.21, n.3, p.251-269, 1973.

GROMYSZ-KALKOWSKA, K. The effect of some exogenous and body weight on oxygen consumption in Glomeris connexa C.L. Koch (Diplopoda). Folia Biol., v.22, n.1, p.37-49, 1974.

GROMYSZ-KALKOWSKA, K. Influence of temperature and duration of aclimation on the metabolic rate in Orthomorpha gracilis C.L. Koch (Diplopoda). Folia Biol., v.24, n.1, p.55-64, 1976a. 
GROMYSZ-KALKOWSKA, K. The oxygen consumption of Polyzonium germanicum Brdt (Diplopoda) in relation to some exogenous and endogenous factors. Folia Biol., v.24, n.4, p.401-415, 1976 b.

GROMYSZ-KALKOWSKA, K. Some properties of the respiratory metabolism of the adult Leptoiulus proximus proximus (Nem.) (Diplopoda). Folia Biol., v.27, n.2, p.129-145, 1979.

GROMYSZ-KALKOWSKA, K., STOJALOWSKA, W. Respiratory metabolism in Orthomorpha gracilis C.L.Koch (Diplopoda) as a function of temperature and body size. Folia Biol., v.14, n.4, p.379-389, 1966.

HALL, F.R., HOLLINGWORTH, R.M. SHANKLAND, D.L. Cyanid tolerance in millipedes: comparison in millipedes and insects. Entomol. News, v.80, n.11, p.277$82,1969$.

HOFFMAN, R.L. Classification of the Diplopoda. Genève (Suisse): Muséum d'Histoire Naturelle (ed.), 1979. 237p.

HOPKIN, S.P., READ, H.J. The biology of millipedes. New York: Oxford University Press, 1992, xiv $+233 p$.

LEVY Jr., N.C. Atividade locomotora circadiana de Pseudonannolene tricolor (Diplopoda, Spirostreptidae). VIII Reunião Anual da FESBE, Caxambu, MG. Resumos, 13.49, 1993, pág. 267.

LEVY Jr., N.C., PENTEADO, C.H.S. Termopreferência, actografia e ritmos metabólicos de Pseudonannolene tricolor (Diplopoda, Spirostreptidae). III Encontro de Pesquisas de Pós-Graduação - Zoologia, IB/UNESP - Rio Claro, SP, Resumos, 1995, pág. 16.

MAC NEILL, S., LAETON, J.H. Annual production and respiration in animal populations. Nature, v.225, p.472-474, 1970.

MEAD, M., GILHODES, J.C. Organization temporalle de l'activité locomotrice chez un animal cavernicole Blaniulus lichtensteini Bröl. (Diplopoda). J. Comp. Physiol., v.90, p.47-52, 1974.

PARK, O. Studies in Nocturnal Ecology. III. Recording apparatus and further analysis of activity rhythm. Ecology, v.16, p.152-163, 1935.

PARK, O., LOCKETT, J.A., MYERS, D.J. Studies in nocturnal ecology with special reference to climax forest. Ecology, v.12, p.709-727, 1931.

PAULPANDIAN, A. A preliminary report on diurnal rhythm in the locomotor activity of pill millipede Arthrosphaera dalyi (Pocock). Proc. Indian Acad. Sci., sec.B, v.62, n.5, 
p.235-241, 1965.

PAULPANDIAN, A. A note on the respiration of the pill millipede Arthrosphaera dalyi (Pocock). Curr. Sci., v.7, p.178-179, 1966.

PEDRO, S.R.M., PENTEADO, C.H.S. Comportamento rítmico do diplópode Rhinocricus varians (Spirobolida, Rhinocricidae) sob fotoperíodo normal e invertido. Cien. Cult., São Paulo, v.38, n.7, p.382, 1986. (Resumos da 38a Reunião Anual da SBPC).

PENTEADO, C.H.S., MENDES, E.G. Respiratory metabolism and tolerance in a tropical millipede, Rhinocricus padbergi VERHOFF, 1938. III. The responses to temperature variations. Rev. Brasil. Biol., v.41, n.3, p.499-509, 1981.

PENTEADO, C.H.S., MENDES, E.G. Perda de peso por dessecação e preferências térmicas no diplópodo Rhinocricus padbergi Verhoeff, 1938. Naturalia, v.7, p.17-28, 1982.

PENTEADO, C.H.S., MENDES, E.G. Respiratory metabolism and tolerance in a tropical millipede, Rhinocricus padbergi Verhoef, 1938. IV. Activity and respiratory rhytms. Rev. Brasil. Biol., v.43, n.4, p. 361-372, 1983.

PHILLIPSON, J. Ecological energetics. London: Edward Arnold Ltd. (ed.), 1966. 57p.

PROSSER, C.L. Physiological variation in animals. Biol. rev. v.30, p.229-262, 1955

PROSSER, C.L. Comparative Animal Physiology: I. Environmental Physiology. 3a.ed. Philadelphia: W.B. Saunders Co., 1973. xxii $+454 p+$ xiv.

PROSSER, C.L. Environmental and metabolic animal physiology. Comparative Animal Physiology, $4^{\text {th }}$ Edition, New York: John Wiley-Liss Inc., 1991.

RAO, K.P., BULLOCK, T.H. $\mathrm{Q}_{10}$ as a function of size and habitat temperature in ppoikiloterms. Amer. Nat., v.88. p.33-44, 1954. RAO, K.P., BULLOCK, T.H. Q 10 as a function of size and habitat temperature in ppoikiloterms. Amer. Nat., v.88. p.33-44, 1954.

REICHLE, D.E. Relation of body size to food intake, oxygen consumption and trace element metabolism in forest floor arthropods. Ecology, v.49, n.3, p.539-542, 1968.

SCHMIDT-NIELSEN, K. Animal physiology: adaptation and environment. 4.ed. New York, Cambridge University Press, 1990, 602 p.

SCHUBART, O. Os diplópodos de Pirassununga. Tucumán (Argentina): Acta Zoológica 
Lilloana del Instituto "Miguel Lillo", tomo II, p.321-440, 1944.

SCHUBART, O. Sôbre os diplopoda dos estados do Paraná e Santa Catarina. I. Proterospermophora. Arq. Mus. Paran., v.10, p.77-132, 1953.

SILVA, R.S. Fisioecologia do diplópodo Oxidus gracilis (Koch, 1847) (Polydesmida, Strongylosomidae): Respostas Comportamentais e Metabólicas. Rio Claro: Instituto de Biociências, UNESP, 1997. vi + 93p. Dissertação (Mestr. Zool. Inv.) - Instituto de Biociências, Universidade Estadual Paulista, 1997.

SOKAL, R.R., ROHLF, F.J. Biometry - The principles and practice of statistics in biological research. San Francisco: W.H. Freeman and Co, 1981. 855p.

STAMOU, G.P., IATROU, G.D. Studies on the respiratory metabolism of Glomeris balcanica (Diplopoda, Glomeridae). J. Insect. Physiol., v.39, n.6, p.529-35, 1993.

STEWART, T.C. Measurement of circadian locomotor activity rhytms in the xystodesmid millipede Pachydesmus crasicustis. Louis. Acad. Sci., v.32, p.40-43, 1969.

STEWART, T.C., WOODRING, J.P. Anatomical and physiological studies of water balance in the millipedes Pachydesmus crasicustis (Polydesmida) and Orthoporus textilulens (Spirobulida). Comp. Biochem. Physiol., v.44A, p.735-60, 1973

STOJALOWSKA, W. Some notes Polydesmus complanatus (L.) under rearing conditions. Ann. Univ. Mariae Curie-Sklodowska, Lublin - Polônia, sec.C, v.15, n.11, p.213-223, 1960.

TOYE, S.A. Studies on the locomotory activity of three species of nigerian millipedes: Spirostreptus assiniensis, Oxydesmus sp and Habrodesmus falx. Ent. exp \& appl., v.9, n.3, p.369-377, 1966c

WERNICK, A.M., PENTEADO, C.H.S, CABRAL, R.A. Metabolismo respiratório de Leptodesmus dentellus Schubart 1946 e Sandalodesmus gasparae Schubart, 1946 (Diplopoda, Polydesmida) em diferentes temperaturas. Naturalia, v.8, p.227-234, 1983.

WIESER, W. Effects of Temperature on Ectothermic Organisms. Berlin: Springer Verlag, New York: Heidelberg, 1973. xi +298 p.

WOOTEN, R.C., CRAWFORD, C.S. Respiratory metabolism of the desert millipede Orthoporus ornatus (Girard) (Diplopoda). Oecologia, v.17, p.179-186, 1974.

WOOTEN, R.C., CRAWFORD, C.S., RIDDLE, W.A. Behavioral thermoregulation of Orthoporus ornatus (Diplopoda, Spirostreptidae) in three desert habitas. Zool. J. 
Linnean Soc., v.57, n.1, p.59-74, 1975.

ZAR, J.H. Biostatistical analysis. Prentice Hall Inc., 1974. 620p. 